\title{
ANÁLISE DA VIBRAÇÃO NO PROCESSAMENTO SECUNDÁRIO DE MADEIRAS COM DIFERENTES MASSAS ESPECÍFICAS APARENTES
}

\author{
Nilton Cesar Fiedler ${ }^{1}$, Heitor Broetto Marin ${ }^{2}$, José Tarcísio da Silva Oliveira ${ }^{1}$, \\ Flávio Cipriano Assis do Carmo ${ }^{3}$ \\ ${ }^{1}$ Eng. Florestal, Dr., Depto. de Engenharia Florestal, UFES, Alegre, ES - nilton.fiedler@ufes.br; jtsilva@npd.ufes.br \\ ${ }^{2}$ Eng. Florestal, UFES, Alegre, ES - heitorbrma@ yahoo.com.br \\ ${ }^{3}$ Eng. Florestal, Mestrando em Ciências Florestais, UFES, Alegre ES - flaviocipriano@ hotmail.com \\ Recebido para publicação: 10/06/2011 - Aceito para publicação: 05/02/2013
}

\begin{abstract}
Resumo
O objetivo desta pesquisa foi identificar os níveis de vibração ocorridos no processamento secundário da madeira e quantificar sua interação com a diferença de massa específica aparente entre as espécies de madeira. A pesquisa foi desenvolvida na marcenaria do Departamento de Ciências Florestais e da Madeira, do Centro de Ciências Agrárias da Universidade Federal do Espírito Santo, localizada no município de Jerônimo Monteiro, sul do Espírito Santo, no período de agosto a dezembro de 2010. As vibrações transmitidas ao sistema mão-braço dos trabalhadores pelas máquinas foram coletadas por intermédio de um acelerômetro e de um medidor de vibração. As espécies utilizadas na pesquisa foram Apuleia leiocarpa, Eucalyptus grandis versus E. urophylla (híbrido urograndis), Corymbia citriodora e Pterogyne nitens. Todas as máquinas apresentaram classificação de conforto para o eixo XY (palma da mão e nós dos dedos) e classificação de fadiga para o eixo Z (punhos). A serra fita foi a única máquina que não apresentou variação da vibração em relação à espécie processada. Conforme a classificação de fadiga para o eixo Z, é necessária a execução de medidas para o controle da vibração. Palavras-chave: Ergonomia florestal; ambiente de trabalho; densidade da madeira; marcenaria.
\end{abstract}

\begin{abstract}
Analysis of vibration in secondary wood processing with different apparent specific masses. The aim was to identify the levels of vibration that occurred in the secondary wood processing, and to quantify vibration interaction with the difference of apparent specific mass. The research was developed in wood workshops of Department of Forestry, Center for Agrarian Sciences, Federal University of Espirito Santo, located in Jerônimo Monteiro city, south of Espírito Santo, from August to December 2010. The vibrations transmitted to the hand-arm system of the workers by the machines had been collected by an accelerometer 20 model NK and a measurer of vibration model NK 300. The species used in the research had been Apuleia leiocarpa, Eucalyptus grandis versus E. urophylla (hybrid urograndis), citriodora Corymbia and Pterogyne nitens. All the machines had presented classification of comfort for axle XY and classification of fatigue for axle $\mathrm{Z}$. As result, according to the classification of fatigue for axle $\mathrm{Z}$, measures are necessary for the control of the vibration.

Keywords: Forest ergonomics; work environment; density of the wood, joinery.
\end{abstract}

\section{INTRODUÇÃO}

As operações de processamento secundário são aquelas realizadas logo após o desdobro principal e visam à redução das dimensões das peças de madeira ou ao dimensionamento final das mesmas, tanto no comprimento quanto na largura e na espessura. Faz parte do cotidiano das indústrias de madeira serrada a utilização de diferentes dimensões de matéria-prima, tornando-se ampla a gama de produtos serrados.

As atividades contempladas no processamento da madeira são desenvolvidas em marcenarias. Segundo Fiedler et al. (2009), essas atividades exigem grande atenção e precisão em sua realização, devido ao maquinário envolvido na confecção dos produtos e ao elevado nível de risco aos quais os operadores estão expostos, ocasionados pelas máquinas e fatores ambientais envolvidos no processo. 
A vibração compreende qualquer movimento que o corpo executa em torno de um ponto fixo. Esse movimento pode ser regular, do tipo senoidal, ou irregular, quando não segue nenhum padrão determinado. Para Santos Filho et al. (2003), as vibrações consistem em uma mistura complexa de diversas ondas, com frequências e direções diferentes. A vibração é um agente nocivo presente em várias atividades do cotidiano do trabalhador (IIDA, 2005).

A transmissão da vibração ao operador acarreta problemas de saúde, como a perda de precisão dos movimentos. O diagnóstico das doenças associadas à vibração ainda não é efetivamente preciso, pois geralmente é distorcido por diagnósticos de esforços repetitivos e não associado aos problemas advindos da utilização das máquinas com manutenção inadequada ou com problemas de fabricação.

A vibração é um fator de difícil exclusão no processo de desenvolvimento e utilização das máquinas, devido ao atrito das peças sujeitas à movimentação para a realização das atividades. Entretanto, deve-se atuar na manutenção preventiva, para evitar folgas entre as peças, contribuindo para o controle da vibração dentro dos limites toleráveis pelo guia de avaliação da exposição humana à vibração, de acordo com a norma ISO 5349-1 de 2001, para uma jornada de trabalho confortável.

Segundo Nunes (2005), na Vibração Mão-Braço (VMB), são transmitidas vibrações mecânicas que, quando transmitidas ao sistema mão-braço do operador, implicam riscos para a saúde e para a segurança dos trabalhadores, em especial perturbações vasculares, lesões ósteo-articulares e perturbações neurológicas ou musculares.

Partindo da hipótese de que o processamento de madeiras com diferentes massas específicas aparentes pode causar níveis diferenciados de vibração nas mãos e braços dos operadores, conforme a máquina utilizada no processamento secundário, esta pesquisa objetivou quantificar os níveis de vibração a que os trabalhadores estão expostos durante o processamento secundário da madeira em diferentes máquinas e sua interação com as diferentes massas específicas aparentes das madeiras utilizadas.

\section{MATERIAL E MÉTODOS}

\section{Área de estudo}

A pesquisa foi desenvolvida durante os meses de agosto a dezembro de 2010 na marcenaria localizada no Departamento de Ciências Florestais e da Madeira do Centro de Ciências Agrárias da Universidade Federal do Espírito Santo, situada no município de Jerônimo Monteiro, sul do estado do Espírito Santo. As espécies utilizadas na pesquisa foram garapa (Apuleia leiocarpa (Vogel) J. F. Macbr.), da família Caesalpiniaceae, eucalipto (Eucalyptus grandis versus E. urophylla - híbrido urograndis), da família Myrtaceae, corimbia citriodora (Corymbia citriodora Hook), da família Myrtaceae, e amendoimbravo (Pterogyne nitens Tull.), da família Caesalpiniaceae.

As peças foram colocadas em uma câmara climática a $25^{\circ} \mathrm{C}$ de temperatura e $60 \%$ de umidade relativa, permanecendo nessa condição até atingirem o equilíbrio higroscópico próximo de $13 \%$ (Tabela 1). Para determinação da massa específica aparente e da umidade das madeiras, foi utilizada a metodologia proposta pela NBR 7190/1997 (Projeto de estruturas de madeira).

Tabela 1. Massa específica aparente e umidade das madeiras.

Table 1. Apparent specific mass and humidity of wood.

\begin{tabular}{lcc}
\hline Espécies & $\begin{array}{c}\text { Massa específica aparente } \\
\left(\mathbf{g} / \mathbf{c m}^{\mathbf{3}}\right)\end{array}$ & Umidade (\%) \\
\hline Apuleia leiocarpa & 0,63 & 11,89 \\
Pterogyne nitens & 0,52 & 14,28 \\
Eucalyptus grandis versus E. urophylla & 0,52 & 12,13 \\
Corymbia citriodora & 0,85 & 14,35 \\
\hline
\end{tabular}

As máquinas utilizadas durante a avaliação da vibração foram: serra circular, serra fita, desempenadeira e desengrossadeira, descritas na tabela 2.

\section{Coleta de dados}

Os dados de vibração foram coletados durante o processamento secundário de cada espécie, segundo as diferenças de massa específica aparente. Utilizou-se um acelerômetro modelo NK 20 e um medidor de vibração NK 300, ambos da marca Teknikao (Figura 1). 
Tabela 2. Descrição técnica das máquinas analisadas.

Table 2. Technical description of the analyzed machines.

\begin{tabular}{|c|c|}
\hline Máquinas & Descrição \\
\hline Serra circular & $\begin{array}{l}\text { Utilizada para serrar madeira ou derivados em cortes retos, por meio } \\
\text { de disco dentado, acoplada em uma mesa de corpo fixo. A serra } \\
\text { utilizada foi da marca Invicta, modelo SCI- } 25 \text {, com potência de } \\
\text { motor de } 2 \mathrm{hp} \text {. Altura de corte de } 65 \mathrm{~mm} \text {, diâmetro de serra de } \\
250 \mathrm{~mm} \text {, inclinação da serra de } 45^{\circ} \text { e rotação da serra de } 3800 \mathrm{rpm} \text {. }\end{array}$ \\
\hline Serra fita & $\begin{array}{l}\text { Utilizada para serrar madeira ou derivados em cortes retos. A } \\
\text { maquina utilizada foi da marca Invicta, modelo SFI- } 80 \text {, diâmetro dos } \\
\text { volantes de } 800 \mathrm{~mm} \text {, largura dos volantes de } 50 \mathrm{~mm} \text {, rotação dos } \\
\text { volantes de } 600 \mathrm{rpm} \text {, comprimento de lâmina variando entre } 5552 \mathrm{e} \\
5762 \mathrm{~mm} \text { e potência do motor trifásico de } 5 \mathrm{cv} \text {. }\end{array}$ \\
\hline Desempenadeira & $\begin{array}{l}\text { Utilizada para plainar a superfície da peça. A máquina utilizada foi } \\
\text { da marca Invicta, modelo DI- } 32 \text {, com rotação do eixo porta-facas de } \\
4375 \mathrm{rpm} \text {, altura máxima de corte de } 19 \mathrm{~mm} \text {, inclinação da guia de } \\
45^{\circ} \text { e potência do motor de } 3 \mathrm{cv} \text {. }\end{array}$ \\
\hline Desengrossadeira & $\begin{array}{l}\text { Visa dimensionar a espessura das peças. É constituída por facas e } \\
\text { dois rolos de alimentação que funcionam automaticamente. A } \\
\text { máquina utilizada foi da marca Invicta, modelo DGC- } 40 \text {, com } \\
\text { rotação do eixo porta-facas de } 5000 \mathrm{rpm} \text {, diâmetro dos rolos de } \\
\text { avanço de } 75 \mathrm{~mm} \text {, altura de corte variando entre } 5 \text { e } 400 \mathrm{~mm} \text {, } \\
\text { velocidade de avanço de } 7,5 \mathrm{~m} / 14 \text { min e potência do motor de } 5 \mathrm{cv} \text {. }\end{array}$ \\
\hline
\end{tabular}

Fonte: Fiedler et al. (2010).

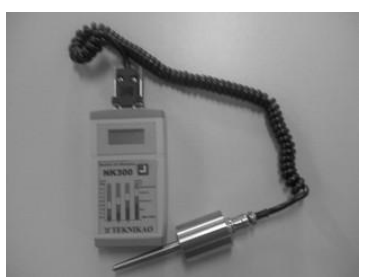

Figura 1. Acelerômetro e medidor de vibração.

Figure 1. Accelerometer and a meter of vibration.

Para a coleta, a vibração foi medida de acordo com as três coordenadas ortogonais definidas na norma ISO 5349-1 (2001) (eixo "xx" - através da palma da mão; eixo "yy" - através dos nós dos dedos; eixo "zz" - paralelo aos ossos do braço), como demonstrado na figura 2. Durante a coleta de dados, após cada ciclo de operação, as máquinas eram desligadas para manutenção e afiação dos gumes de corte, para que todos os corpos de prova sofressem as mesmas condições de processamento. A velocidade de avanço era de $7,5 \mathrm{~m} /$ minuto e a velocidade de corte durante o processamento era a mesma para todos os corpos de prova analisados.
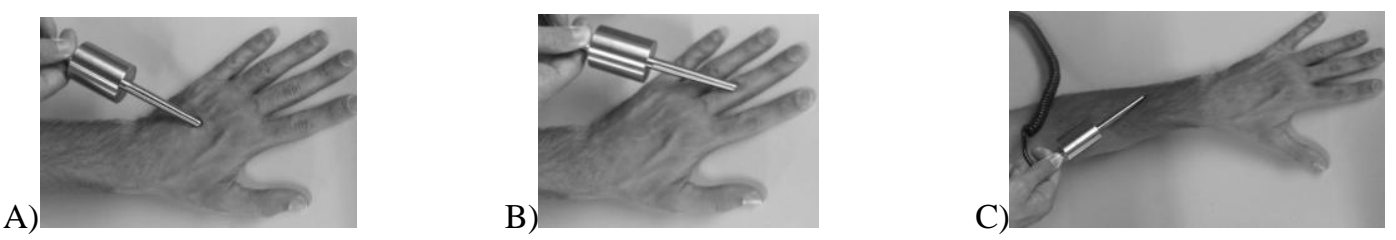

Figura 2. Coordenadas ortogonais ISO 5349-1 (A: eixo xx, B: eixo yy e C: eixo zz).

Figure 2. Coordinated ortogonais ISO 5349-1 (A: axis xx, B: axis yy and C: axis zz).

Inicialmente foi feito um estudo piloto para definir o tamanho mínimo da amostra de dados a serem utilizados na pesquisa. Esses dados foram analisados por meio da expressão proposta por Conaw (1977): 


$$
\mathrm{n} \geq \frac{\mathrm{t}^{2} * \mathrm{~s}^{2}}{\mathrm{e}^{2}}
$$

Equação 1

Em que: $n=$ número de amostras ou pessoas necessárias;

$\mathrm{t}=$ valor tabelado a $10 \%$ de probabilidade (distribuição t de Student);

$\mathrm{s}=$ desvio padrão da amostra;

$\mathrm{e}=$ erro admissível a $10 \%$.

A avaliação do nível de exposição baseou-se no cálculo do valor da exposição diária para um período de referência de 8 horas $(A(8))$. Por meio da ISO 2631, de 1978, foram utilizados os critérios de severidade para adequação dos parâmetros de classificação da vibração mão-braço. Os parâmetros são divididos nos eixos $\mathrm{X}, \mathrm{Y}$ e Z, referentes à palma da mão, nós dos dedos e braços, respectivamente.

Para o cálculo da vibração nos eixos XY, utilizou-se a seguinte expressão, definida na norma ISO 5349-1 (2001) e explicada pela EU Good Practice Guide HAV (2006), seguindo as orientações da Directive 2002/44/EC da União Europeia:

$$
A(8)=\sqrt{a_{h w x}^{2}+a_{h w y}^{2}} \quad \text { Equação } 2
$$

Em que: $a_{\text {hwx }}$, a são os valores eficazes (coletados) da aceleração ponderada em freqüência, para a palma da mão e nós dos dedos, respectivamente. Os valores considerados para avaliação do risco são mostrados na tabela 3 .

Tabela 3. Guia para a avaliação da exposição humana à vibração (ISO 5349-1, 2001).

Table 3. Guideline to evaluation of human exposition to vibration (ISO 5349-1, 2001).

\begin{tabular}{lcc}
\hline Critérios de severidade & Aceleração $(\mathbf{X}, \mathbf{Y})$ horizontal $\left(\mathbf{m} / \mathbf{s}^{\mathbf{2}}\right)$ & Aceleração $(\mathbf{Z})$ vertical $\left(\mathbf{m} / \mathbf{s}^{\mathbf{2}}\right)$ \\
\hline Exposição & 0,448 & 0,630 \\
Fadiga & 0,224 & 0,315 \\
Conforto & 0,071 & 0,100 \\
\hline
\end{tabular}

Tabela 4. Espécies e máquinas avaliadas, número mínimo e coletado de amostras.

Table 4. Species and machinery evaluated, minimum number of samples, and number of collected samples.

\begin{tabular}{lccc}
\hline Espécies & Máquinas & $\mathbf{N}^{\mathbf{0}}$ mínimo de amostras & $\mathbf{N}^{\mathbf{0}}$ de amostras coletadas \\
\hline Pterogyne nitens & Serra circular & 18 & 24 \\
& Serra fita & 15 & 29 \\
& Desempenadeira & 27 & 29 \\
& Desengrossadeira & 11 & 14 \\
\hline Eucalyptus grandis versus & Serra circular & 14 & 23 \\
E. urophylla & Serra fita & 15 & 23 \\
& Desempenadeira & 20 & 22 \\
& Desengrossadeira & 14 & 16 \\
\hline Apuleia leiocarpa & Serra circular & 20 & 22 \\
& Serra fita & 12 & 22 \\
& Desempenadeira & 7 & 26 \\
& Desengrossadeira & 15 & 17 \\
\hline Corymbia citriodora & Serra circular & 12 & 30 \\
& Serra fita & 12 & 28 \\
& Desempenadeira & 11 & 25 \\
& Desengrossadeira & 12 & 18 \\
\hline
\end{tabular}

Na tabela 4 estão descritos os dados coletados em relação ao número de amostras ideais para um nível mínimo aceitável ao nível de $10 \%$ de significância. 


\section{RESULTADOS E DISCUSSÕES}

A classificação dos níveis de conforto nas máquinas, conforme médias encontradas de vibração nas coordenadas XY e Z, são apresentadas na tabela 5 .

Tabela 5. Classificação dos níveis de conforto nas máquinas conforme a madeira processada.

Table 5. Classification of comfort levels of machines regarding processed wood.

\begin{tabular}{|c|c|c|c|c|}
\hline Espécies & Máquinas & Coordenadas & Média & Classificação \\
\hline \multirow[t]{8}{*}{ Pterogyne nitens } & Serra circular & $\mathrm{XY}$ & 0,042 & Conforto \\
\hline & & $\mathrm{Z}$ & 0,163 & Fadiga \\
\hline & Serra fita & $X Y$ & 0,035 & Conforto \\
\hline & & $\mathrm{Z}$ & 0,157 & Fadiga \\
\hline & Desempenadeira & $\mathrm{XY}$ & 0,051 & Conforto \\
\hline & & $\mathrm{Z}$ & 0,169 & Fadiga \\
\hline & Desengrossadeira & $X Y$ & 0,054 & Conforto \\
\hline & & $\mathrm{Z}$ & 0,174 & Fadiga \\
\hline \multirow{8}{*}{$\begin{array}{l}\text { Eucalyptus grandis versus } \\
\text { E. urophylla }\end{array}$} & Serra circular & $\mathrm{XY}$ & 0,041 & Conforto \\
\hline & & $\mathrm{Z}$ & 0,158 & Fadiga \\
\hline & Serra fita & $\mathrm{XY}$ & 0,037 & Conforto \\
\hline & & $\mathrm{Z}$ & 0,146 & Fadiga \\
\hline & Desempenadeira & $X Y$ & 0,055 & Conforto \\
\hline & & $\mathrm{Z}$ & 0,178 & Fadiga \\
\hline & Desengrossadeira & $X Y$ & 0,057 & Conforto \\
\hline & & $\mathrm{Z}$ & 0,186 & Fadiga \\
\hline \multirow[t]{8}{*}{ Apuleia leiocarpa } & Serra circular & $\mathrm{XY}$ & 0,045 & Conforto \\
\hline & & $\mathrm{Z}$ & 0,171 & Fadiga \\
\hline & Serra fita & $X Y$ & 0,033 & Conforto \\
\hline & & $\mathrm{Z}$ & 0,143 & Fadiga \\
\hline & Desempenadeira & $X Y$ & 0,048 & Conforto \\
\hline & & $\mathrm{Z}$ & 0,173 & Fadiga \\
\hline & Desengrossadeira & $X Y$ & 0,050 & Conforto \\
\hline & & $\mathrm{Z}$ & 0,173 & Fadiga \\
\hline \multirow[t]{8}{*}{ Corymbia citriodora } & Serra circular & $\mathrm{XY}$ & 0,045 & Conforto \\
\hline & & $\mathrm{Z}$ & 0,174 & Fadiga \\
\hline & Serra fita & $X Y$ & 0,040 & Conforto \\
\hline & & $\mathrm{Z}$ & 0,154 & Fadiga \\
\hline & Desempenadeira & $X Y$ & 0,049 & Conforto \\
\hline & & $\mathrm{Z}$ & 0,172 & Fadiga \\
\hline & Desengrossadeira & $X Y$ & 0,052 & Conforto \\
\hline & & $\mathrm{Z}$ & 0,178 & Fadiga \\
\hline
\end{tabular}

$\mathrm{Na}$ avaliação das massas específicas aparentes e das máquinas, observa-se que, para o eixo Z, todas as máquinas apresentaram como classificação a fadiga. Esses resultados são semelhantes aos encontrados por Fiedler et al. (2010) em avaliação subjetiva de vibração em marcenarias, em que 64\% dos operadores afirmaram haver vibração excessiva, causando dores principalmente nos punhos.

A maior vibração no eixo $\mathrm{Z}$ se refere ao fato de que o operador concentra os maiores esforços nas articulações dos punhos, ou seja, aplica constantemente repetições de movimentos no eixo Z. Com o decorrer do tempo de trabalho nessas atividades, o trabalhador poderá ficar sujeito a várias doenças do trabalho. Independentemente do tipo de madeira utilizada, é necessária uma intervenção urgente na maneira como se faz o processamento secundário das madeiras utilizadas na fabricação de móveis. Para o eixo XY, todas as máquinas apresentaram a classificação de conforto para todas as variações de massa específica.

A figura 3 mostra que, para a aceleração horizontal $X Y\left(\mathrm{~m} / \mathrm{s}^{2}\right)$, todas as máquinas obtiveram valores menores do que o limite aceitável de conforto (ISO 5349-1, 2001). Já para a aceleração vertical Z, 
as diferentes espécies para todas as máquinas apresentaram valores superiores ao nível aceitável de conforto.

\section{Aceleração XY}

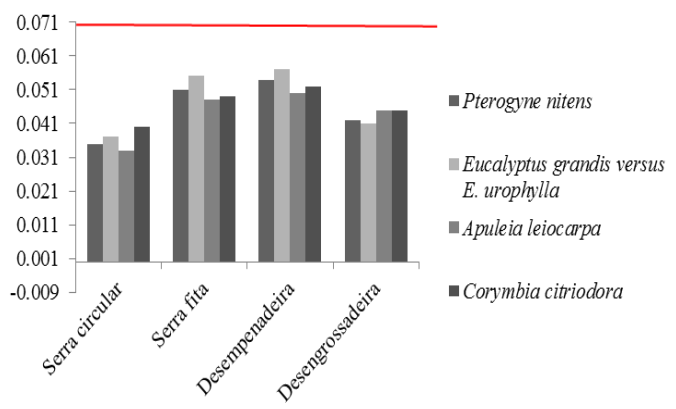

\section{Aceleração Z}

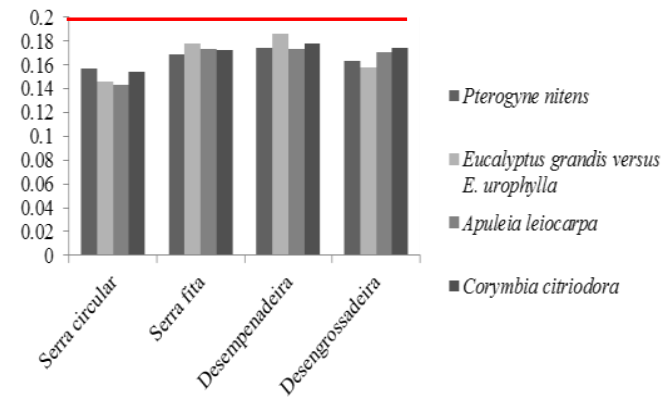

Figura 3. Distribuição dos níveis de vibração das máquinas em relação às espécies analisadas.

Figure 3. Distribution of vibration levels of machines in relation to the analyzed species.

Na tabela 6 são apresentados os resultados da variação das espécies de diferentes massas específicas aparentes em relação ao fator fixo máquinas.

Tabela 6 Resultados do teste de comparações de média pelo teste de Tukey para a variável vibração em função da relação das máquinas pela massa específica aparente das madeiras a $10 \%$ de significância.

Table 6. Results of the average comparison test by the test of Tukey for the variable vibration in function of the relation of the machines by the apparent specific mass of wood at $10 \%$ of significance.

\begin{tabular}{lcccc}
\hline $\begin{array}{l}\text { Espécie } \\
\left.\text { (massa específica aparente }-\mathbf{g} / \mathbf{c m}^{3}\right)\end{array}$ & \multicolumn{4}{c}{ Máquinas } \\
\cline { 2 - 5 } Corymbia citriodora $(0,85)$ & $0,065 \mathrm{a}$ & $0,056 \mathrm{a}$ & $0,067 \mathrm{a}$ & $0,067 \mathrm{ab}$ \\
Eucalyptus grandis versus & $0,059 \mathrm{a}$ & $0,053 \mathrm{ab}$ & $0,073 \mathrm{ab}$ & $0,072 \mathrm{a}$ \\
E. urophylla $(0,52)$ & $0,058 \mathrm{a}$ & $0,053 \mathrm{ab}$ & $0,074 \mathrm{ab}$ & $0,063 \mathrm{~b}$ \\
Pterogyne nitens $(0,52)$ & $0,063 \mathrm{a}$ & $0,048 \mathrm{~b}$ & $0,065 \mathrm{~b}$ & $0,065 \mathrm{ab}$ \\
Apuleia leiocarpa $(0,63)$ & &
\end{tabular}

A serra fita e a desempenadeira apresentaram os mesmos resultados, sendo possível observar que as massas específicas aparentes mais elevadas se igualaram às menores e diferiram estatisticamente entre si pelo teste de Tukey a 10\% de significância. Para a desengrossadeira, houve similaridade entre as madeiras de maior massa específica aparente, entretanto cada uma em um grupo diferente. Já para a serra circular, a massa específica aparente da madeira não diferiu estatisticamente para o índice médio de vibração.

As espécies de menores massas específicas aparentes apresentaram resultados diferentes somente para a desengrossadeira, enquanto as espécies de maiores massas específicas aparentes apresentaram resultados iguais para todas as máquinas.

$\mathrm{Na}$ tabela 7 são apresentados os resultados ao se fixarem as espécies em relação à variável máquina.

Para a espécie Pterogyne nitens, a desempenadeira apresentou maior valor médio de vibração que as demais, enquanto a serra fita e a serra circular apresentaram os menores valores médios entre as máquinas. Na espécie Eucalyptus grandis versus E. urophylla, houve um mesmo valor médio entre a desempenadeira e desengrossadeira, diferindo da serra circular e serra fita, que se igualaram estatisticamente pelo teste de Tukey a $10 \%$ de significância. Já nas espécies de massa específica aparente mais elevada, ocorreu uma similaridade dos resultados. Para Apuleia leiocarpa e Corymbia citriodora, somente a serra fita diferiu das demais máquinas.

Analisando a tabela 5 de classificação qualitativa, observa-se que a massa específica aparente da madeira não influenciou significativamente a vibração, conforme o índice de conforto encontrado em 
todas as repetições. Fazendo a inferência das tabelas 6 e 7, conforme a estatística apresentada, é observado que a massa específica aparente da madeira pouco difere estatisticamente entre as madeiras e suas interações com as máquinas.

Tabela 7. Resultados do teste de comparações de média pelo teste de Tukey para a variável vibração em relação à massa específica aparente das madeiras através do uso em diferentes máquinas a 10\% de significância.

Table 7. Results of the average comparison test by the test of Tukey for the variable vibration generated from the relation of the apparent specific mass of wood by the use in different machines at $10 \%$ of significance.

\begin{tabular}{|c|c|c|c|c|}
\hline \multirow[b]{2}{*}{ Máquinas } & \multicolumn{4}{|c|}{ Espécie (massa específica aparente $\mathbf{g} / \mathrm{cm}^{3}$ ) } \\
\hline & $\begin{array}{c}\text { Pterogyne nitens } \\
(0,52)\end{array}$ & $\begin{array}{c}\text { Eucalyptus grandis } \\
\text { versus } E \text {. urophylla } \\
(\mathbf{0 , 5 2 )}\end{array}$ & $\begin{array}{c}\text { Apuleia leiocarpa } \\
(\mathbf{0 , 6 3 )}\end{array}$ & $\begin{array}{c}\text { Corymbia citriodora } \\
(\mathbf{0 , 8 5})\end{array}$ \\
\hline Serra circular & $0,058 \mathrm{bc}$ & $0,059 \mathrm{~b}$ & $0,063 \mathrm{a}$ & $0,065 \mathrm{a}$ \\
\hline Serra fita & $0,053 \mathrm{c}$ & $0,053 \mathrm{~b}$ & $0,048 \mathrm{~b}$ & $0,056 \mathrm{~b}$ \\
\hline Desempenadeira & $0,074 \mathrm{a}$ & $0,073 \mathrm{a}$ & $0,065 \mathrm{a}$ & $0,067 \mathrm{a}$ \\
\hline Desengrossadeira & $0,063 \mathrm{~b}$ & $0,072 \mathrm{a}$ & $0,065 \mathrm{a}$ & $0,067 \mathrm{a}$ \\
\hline
\end{tabular}

O fator vibração no sistema mão-braço pode estar mais bem relacionado com a afiação das lâminas, manutenção das máquinas e seus projetos de desenvolvimento, assim como as propriedades das espécies. Quando observada em valores médios, a serra fita é apresentada com valores inferiores às demais máquinas, fato que pode ser explicado pelo baixo esforço realizado na operação. Os maiores valores encontrados na desengrossadeira, provavelmente, foram acarretados pela maior pressão exercida pelo sistema mão-braço para o acabamento da peça e pela necessidade da maior proximidade de contato com a fonte de vibração.

\section{CONCLUSÕES}

De acordo com os resultados apresentados nesta condição de estudo, conclui-se que:

- A hipótese de que madeiras de diferentes massas específicas aparentes, com o mesmo teor de umidade, causam níveis diferenciados de vibração nas mãos e braços dos operadores conforme a máquina utilizada no processamento secundário foi confirmada pela pesquisa.

- Todas as máquinas apresentaram classificação de conforto para o eixo XY (palma da mão e nós dos dedos) e classificação de fadiga para o eixo $\mathrm{Z}$ (punhos).

- A serra fita foi a única máquina em que a vibração não variou em função da espécie processada, devido ao fato de a máquina apresentar uma potência elevada, assim como sua rotação ser muito alta. A baixa variabilidade nas densidades das madeiras não afeta significativamente a vibração no processamento;

- Conforme a classificação de fadiga nos punhos dos operadores, é necessária a execução de medidas para o controle da vibração no eixo $Z$, bem como adoção de luvas antivibratórias ou troca de máquinas por outras que apresentem projetos construtivos com menor vibração, além da necessidade de sua manutenção periódica.

\section{REFERÊNCIAS}

ASSOCIAÇÃO BRASILEIRA DE NORMAS TÉCNICAS (ABNT). MB-26/40: ensaios físicos e mecânicos da madeira. Rio de Janeiro: 1940. 16 p.

ASSOCIAÇÃO BRASILEIRA DE NORMAS TÉCNICAS (ABNT). NBR 7190/1997 - Projeto de estruturas de madeiras. Rio de Janeiro, 1997. 107 p.

CONAW, P. L. Estatística. São Paulo: Edgard Blucher, 1977. 264 p. 
DIRECTIVE 2002/44/CE Prescrições mínimas de segurança e de saúde relativas à exposição dos trabalhadores aos riscos por agentes físicos (vibrações) do Parlamento Europeu. EU Good Practice Guide HAV; WBV Good practice Guide v. 6, 7 p., 12/06/2006.

FIEDLER, N. C.; OLIVEIRA, J. T. S.; GUIMARÃES, P. P.; ALVES, R. T.; WANDERLEY, F. B.; OLIVEIRA, J. G. L.; MORA, R. Influência da massa específica aparente da madeira no ruído produzido durante o processamento secundário: estudo de caso. Revista Floresta. PR, v. 39, n. 2, p. 401 - 408, 2009.

FIEDLER, N. C.; WANDERLEY, F. B.; NOGUEIRA, M.; OLIVEIRA, J. T. S.; GUIMARÃES, P. P.; ALVES, R. T. Otimização do layout de marcenarias no sul do Espírito Santo, baseado em parâmetros ergonômicos e de produtividade. Revista Árvore, v. 33, n. 1, p. 161 - 170, 2009.

FIEDLER, N. C.; GUIMARÃES, P. P.; ALVES, R. T.; WANDERLEY, F. B. Avaliação ergonômica do ambiente de trabalho em marcenarias no sul do Espírito Santo. Revista Árvore, v. 34, n. 5, p. 907 - 915. 2010.

IIDA, I. Ergonomia: projeto e produção. 2. ed. revisada e ampliada. Editora Edgard Blucher, São Paulo, 2005. $630 \mathrm{p}$.

ISO guide for the evaluation of human exposure to wholebody vibration. ISO 2631 - 1978. ISO, Geneva, 1978. $15 \mathrm{p}$.

NUNES, I. L. A importância da avaliação de factores de risco ocupacionais em políticas de aquisição de equipamentos. O caso da exposição à vibração. Proceedings das $3^{\mathbf{a}}$ Jornadas Técnicas de Engenharia Logística Militar - O ciclo de vida dos materiais, organizada pelo Exército Português, Paço de Arcos, CDRom, 6 de Dezembro (2005). 